\title{
An Improved Analysis of SRPT Scheduling Algorithm on the Basis of Functional Optimization*
}

\author{
Hiroshi Fujiwara Yoshiyuki Sekiguchi
}

\begin{abstract}
The competitive performance of the SRPT scheduling algorithm has been open for a long time except for being 2-competitive, where the objective is to minimize the total completion time. Chung et al. proved that the SRPT algorithm is 1.857-competitive. In this paper we improve their analysis and show a 1.792-competitiveness. We clearly mention that our result is not the best so far, since Sitters recently proved the algorithm is 1.250-competitive. Nevertheless, it is still well worth reporting our analytical method; our analysis is based on the modern functional optimization, which can scarcely be found in the literature on the analysis of algorithms. Our aim is to illustrate the potentiality of functional optimization with a concrete application.
\end{abstract}

Keywords: Analysis of Algorithms; On-line Algorithms; Scheduling; Competitive Analysis; Functional Analysis

\section{Introduction}

The SRPT algorithm is a simple algorithm for online job scheduling to minimize the total completion time. Despite its simplicity, the theoretical performance has been open for a long time, except for being 2-competitive [PSW98]. Breaking the barrier of 2, Chung et al. [CNS10] proved that the SRPT algorithm is 1.857-competitive. The proof was done by using a probabilistic method in which the choice of a probabilistic distribution influences the resulting competitiveness. In this paper we obtain an optimal distribution and consequently show a 1.792competitiveness. More precisely, we formulate a linear program over a function space and find a solution which satisfies the optimality condition.

We clearly mention that our result is not the best so far; Sitters [Sit10] recently proved by a quite different argument that the SRPT algorithm is 1.250-competitive. Nevertheless, it is still well worth reporting our analytical method. Although the analysis of algorithms by means of functional optimization is of great potentiality, its application can scarcely be found in the literature.

Optimization theory has played a significant role in the design and analysis of algorithms. Polynomial-time algorithms for LP or SDP are often embedded as a subroutine. Duality theory is comprehensively applied to performance analysis. In most of the researches, however, the problem to be solved is an optimization over finite-dimensional vector space. To the best of our knowledge, merely the work [FIS11] dealt with a function space, which is regarded as an infinite-dimensional vector space, as we do in this paper.

\footnotetext{
*This work was supported by KAKENHI (19700015, 23700014, 23500014, 19740059, and 22740057).
} 


\section{Preliminaries for the Functional Analysis}

We assume all integrals appearing in this paper to be the Lebesgue integrals. $L^{1}[0,1]$ denotes the set of Lebesgue measurable functions $f:[0,1] \rightarrow \mathbb{R}$ for which $\int_{0}^{1}|f(x)| d x<\infty$. $L^{\infty}[0,1]$ stands for the set of Lebesgue measurable functions $f:[0,1] \rightarrow \mathbb{R}$ whose image is bounded except on a set of measure zero. Indeed, we will only handle functions whose image is simply bounded. We denote by $C[0,1]$ and $\mathrm{ND}[0,1]$ the set of continuous and nondecreasing functions on $[0,1]$, respectively.

We also employ the Lebesgue-Stieltjes integral for dealing with a probabilistic distribution which may not have a density function. What should be noted is just the treatment of a discontinuous point: Let $F \in \mathrm{ND}[0,1]$ that is discontinuous at $c \in[0,1]$ and differentiable elsewhere, and $g \in C[0,1]$. Denoting the derivative of $F$ by $f$, we calculate

$$
\int_{0}^{1} g(x) d F(x)=\int_{0}^{c} g(x) f(x) d x+\int_{c}^{1} g(x) f(x) d x+g(c)(F(c+)-F(c-)),
$$

where $F(c+)$ and $F(c-)$ stand for $\lim _{\epsilon \rightarrow+0} F(c+\epsilon)$ and $\lim _{\epsilon \rightarrow+0} F(c-\epsilon)$, respectively.

The purpose of the Lebesgue integral and the above function spaces is to rigorously specify a space to which a dual variable in an optimization problem belongs. It is well known that dual variables that correspond to a finite set of constraints form $\mathbb{R}^{n}$. Concerning a constraint that holds true over a real interval, however, a more careful and involved argument is required. Although definition of such space is rather intricate, resulting functions in this paper are all elementary. Therefore, any integral of a function given in the explicit form may be considered as the Riemann integral. As for the Lebesgue integral and function spaces, refer to [Roy88, Lue69, RS90].

\section{Formulation and Observation}

Chung et al. [CNS10] studied the performance of the SRPT algorithm for online preemptive job scheduling on identical parallel machines, where the objective is to minimize the total completion time. Here, the SRPT algorithm always executes the jobs with shortest remaining processing time. Although the SRPT algorithm schedules deterministically, the following lemma evaluates the competitive performance based on a probabilistic argument.

Lemma 1. ([CNS10]) Let $X$ be a random variable on the interval $[0,1]$. Then the competitive ratio of SRPT is at most

$$
E[X]+\max _{0 \leq a \leq 1} \frac{1}{1+a}(\operatorname{Pr}[0 \leq X \leq a]+\operatorname{Pr}[a<X \leq 1] E[X \mid a<X \leq 1])+1 .
$$

Chung et al. applied the specific cumulative distribution $F(x)=\operatorname{Pr}[X \leq x]=1-(1-x)^{7}$.

Theorem 1. ([CNS10]) The SRPT algorithm is 1.857-competitive.

We find an optimal distribution $F_{0}(x)=1-\frac{1}{1+\gamma_{0}} \ln \frac{1-x}{1-\gamma_{0}}$ for $0 \leq x \leq \gamma_{0}$ and 1 for $\gamma_{0}<x \leq 1$ by the following argument, and obtain the next theorem, where $\gamma_{0} \approx 0.442$.

Theorem 2. The SRPT algorithm is 1.792-competitive, which is the best possible on the basis of Lemma 1.

As we have mentioned before, our result is not the best so far. 
Theorem 3. ([Sit10]) The SRPT algorithm is 1.250-competitive.

We begin with formulating a functional optimization problem to find a distribution which minimizes (1). The set of cumulative distributions $F(x)=\operatorname{Pr}[X \leq x]$ on $[0,1]$ can be identified with $\mathrm{ND}[0,1]$. For $F \in \mathrm{ND}[0,1]$, we define

$$
\begin{aligned}
J(F) & =E[X]=\int_{0}^{1} x d F(x) ; \\
A(F) & =\operatorname{Pr}[0 \leq X \leq 1]=\int_{0}^{1} d F(x) ; \\
B(F)(a) & =\frac{1}{1+a}(\operatorname{Pr}[0 \leq X \leq a]+\operatorname{Pr}[a<X \leq 1] E[X \mid a<X \leq 1]) \\
& =\frac{1}{1+a}\left(\int_{0}^{a} d F(x)+\int_{a}^{1} x d F(x)\right), a \in[0,1] .
\end{aligned}
$$

Then the problem is a linear functional optimization problem:

$$
\begin{aligned}
(\mathcal{P}) \quad \operatorname{minimize} & J(F)+\beta \\
\text { subject to } & A(F)=1 \\
& B(F)(a) \leq \beta, a \in[0,1] \\
& F \in \mathrm{ND}[0,1] .
\end{aligned}
$$

Unlike classical functional problems that can be solved by the calculus of variations, our problem involves optimization with the modern functional analysis since it includes the inequality constraint (3) over a real interval.

It is easily seen that $J: \mathrm{ND}[0,1] \rightarrow \mathbb{R}, A: \mathrm{ND}[0,1] \rightarrow \mathbb{R}$ are linear operators. Here we should specify the whole set of $B(F)$. We first determine the image space of the linear operator $B$. The mapping $a \mapsto B(F)(a)$ is measurable, since the mappings $a \mapsto \int_{0}^{a} d F(x), a \mapsto \int_{a}^{1} x d F(x)$, and $a \mapsto \frac{1}{1+a}$ are monotonic and therefore measurable. In addition, we have

$$
\begin{aligned}
|B(F)(a)| & \leq \frac{1}{1+a}\left(\left|\int_{0}^{a} d F(x)\right|+\left|\int_{a}^{1} x d F(x)\right|\right) \\
& \leq \frac{1}{1+a}\left(\int_{0}^{1} d F(x)+\int_{0}^{1} x d F(x)\right) .
\end{aligned}
$$

Thus $B(F) \in L^{\infty}[0,1]$ for each $F \in \mathrm{ND}[0,1]$.

The rest of this paper is dedicated to solving $(\mathcal{P})$. The sketch is as follows. First, we derive a sufficient condition for optimality. Unfortunately, it is difficult to find a solution which satisfies the condition in a straightforward manner. Thus, we next formulate a subproblem $(\mathcal{Q})$ over a narrowed solution space, with the help of the guess of a solution. $(\mathcal{Q})$ is solved analytically. Finally, we confirm that the obtained solution is also optimal for $(\mathcal{P})$. 


\section{Sufficient Condition for Optimality}

Lemma 2. Let $(\bar{F}, \bar{\beta})$ be feasible to $(\mathcal{P})$. Suppose that there exist $\lambda \in \mathbb{R}, \mu \in L^{1}[0,1]$, and $\nu \in C[0,1]$ satisfying the following conditions:

$$
-J(H)-\eta=\lambda A(H)+\int_{0}^{1} \mu(x)\{B(H)(x)-\eta\} d x-\int_{0}^{1} \nu(x) d H(x), \forall \eta \in \mathbb{R}, \forall H \in \mathrm{ND}[0,1] ;
$$

$\int_{0}^{1} \mu(x)(B(\bar{F})(x)-\bar{\beta}) d x=0$

$\int_{0}^{1} \nu(x) d \bar{F}(x)=0$

$\mu(x) \geq 0, \nu(x) \geq 0, x \in[0,1]$.

Then $(\bar{F}, \bar{\beta})$ is an optimal solution to $(\mathcal{P})$.

Proof. Assume that $\lambda, \mu$, and $\nu$ satisfy the conditions. Then we have

$$
\begin{aligned}
J(\bar{F})+\bar{\beta} & =-\lambda A(\bar{F})-\int_{0}^{1} \mu(x)\{B(\bar{F})(x)-\bar{\beta}\} d x+\int_{0}^{1} \nu(x) d \bar{F}(x) \\
& =-\lambda .
\end{aligned}
$$

Let $F$ be an arbitrary feasible solution to the problem $(\mathcal{P})$. Since $F$ satisfies the constraints of $(\mathcal{P})$ and $\mu(x) \geq 0, \nu(x) \geq 0$, we have

$$
\begin{aligned}
& A(F)=1 \\
& \int_{0}^{1} \mu(x) B(F)(x) d x \leq \int_{0}^{1} \mu(x) \beta d x ; \\
& \int_{0}^{1} \nu(x) d F(x) \geq 0
\end{aligned}
$$

and hence

$$
\begin{aligned}
J(F)+\beta & =-\lambda A(F)-\int_{0}^{1} \mu(x)\{B(F)(x)-\beta\} d x+\int_{0}^{1} \nu(x) d F(x) \\
& \geq-\lambda \\
& =J(\bar{F})+\bar{\beta} .
\end{aligned}
$$

The above argument is similar to a proof of the optimality condition for finite-dimensional linear programming, referred to as the complementary slackness conditions. A different point is that a dual variable corresponding to a constraint over a real interval is a function. The inner product of such a dual variable and a constraint is represented as an integral. Here $\lambda, \mu$, and $\nu$ are dual variables for the constraints (2), (3), and (4), respectively.

The essential difficulty is the proper choice of function spaces. In the discussion below, we will find concrete functions $\mu$ and $\nu$ satisfying the conditions to apply the lemma. So, it is preferable that we choose the function spaces of $\mu$ and $\nu$ as large as possible. On the other hand, we need to restrict the function spaces of $\mu$ and $\nu$ to guarantee finiteness of the integrals appearing in the problem; $\int \mu(x) B(F) d x$ and $\int \nu(x) d F(x)$ have to be finite for all $F \in \mathrm{ND}[0,1]$. 
Here we use the concept of dual function spaces which are the set of all continuous linear functionals defined on the respective spaces.

Now $B(F)$ and $F$ are contained in $L^{\infty}[0,1]$ and $\mathrm{ND}[0,1]$ respectively. It is well known in functional analysis [RS90] that the dual function space of $L^{1}[0,1]$ is $L^{\infty}[0,1]$ and that of $C[0,1]$ is the space of bounded variations which contains $\mathrm{ND}[0,1]$. Thus, we choose $L^{1}[0,1]$ and $C[0,1]$ as the spaces to which $\mu$ and $\nu$ belong, respectively. Then the function spaces are large enough and all integrations are defined and finite. Note that then it is easier to find $\mu$ since it can have discontinuous points. However, the lemma says that we have to find $\nu$ among continuous functions.

\section{Guess of a Solution}

It seems difficult to derive a solution to the problem $(\mathcal{P})$ simply by manipulating the equations and inequalities in Lemma 2. In what follows we construct a subproblem which is easier to handle.

We guess a candidate for a solution making some assumptions. First, let us assume that there exists a density function $f=\frac{d F}{d x}$ and that the equality sign of $(3)$ in $(\mathcal{P})$ holds for all $a \in[0,1]$. Then, we have a differential equation and obtain $f(a)=\frac{\beta}{1-a}$ as a solution to it. We see, however, that while $\int_{0}^{y} f(a) d a$ remains finite for $0<y<1, \int_{0}^{1} f(a) d a$ diverges. Therefore, it turns out that the equality sign of (3) does not hold for an interval including the point one.

Next, suppose that $F$ is discontinuous at a point $b \in(0,1)$ and denote $l=\lim _{x \rightarrow b+0} F(x)-$ $\lim _{x \rightarrow b-0} F(x)(>0)$. In the following we show that the jump can be moved to the point zero without increasing the objective function. Set $\tilde{F} \in \mathrm{ND}[0,1]$ as: $\tilde{F}(x)=F(x)+l$ for $x \in[0, b)$ and $\tilde{F}(x)=F(x)$ for $x \in[b, 1]$. Then, we have $J(\tilde{F})=J(F)-b l<J(F)$ and $A(\tilde{F})=A(F)$. Also, $B(\tilde{F})(a)=B(F)(a)-\frac{b l}{1+a}<B(F)(a)$ for $a \in[0, b)$ and $B(\tilde{F})(a)=B(F)(a)$ for $a \in[b, 1]$. That is to say, if $F$ is feasible, then $\tilde{F}$ is also feasible. The value of the objective function for $\tilde{F}$ is smaller than that for $F$. Hence, it suffices to consider functions which are possibly discontinuous at the point zero and continuous elsewhere.

Through these observations we guess that: (I) There exists a density function $f$ on $(0,1)$. (II) The equality sign of (3) holds for a certain interval $(0, \gamma]$. (III) $f(x)=0$ for $x \in(\gamma, 1)$. (IV) $F$ is possibly discontinuous at zero.

We formulate a subproblem $(\mathcal{Q})$ over the narrowed solution space. For the sake of convenience, we define $f(0)=f(1)=0$ and $\lim _{x \rightarrow-0} F(x)=0$. An integral is thus calculated as

$$
\int_{0}^{t} g(x) d F(x)=g(0) F(0)+\int_{0}^{t} g(x) f(x) d x
$$

for $0<t \leq 1$.

$$
\begin{aligned}
& (\mathcal{Q}) \text { minimize } \int_{0}^{\gamma} x f(x) d x+\beta \\
& \text { subject to } F(0)+\int_{0}^{\gamma} f(x) d x-1=0 \\
& \frac{1}{1+a}\left(F(0)+\int_{0}^{a} f(x) d x+\int_{a}^{\gamma} x f(x) d x\right)-\beta=0, a \in(0, \gamma] \\
& f(a)=0, a \in(\gamma, 1] .
\end{aligned}
$$

An optimal solution is analytically derived. See Figure 1. 


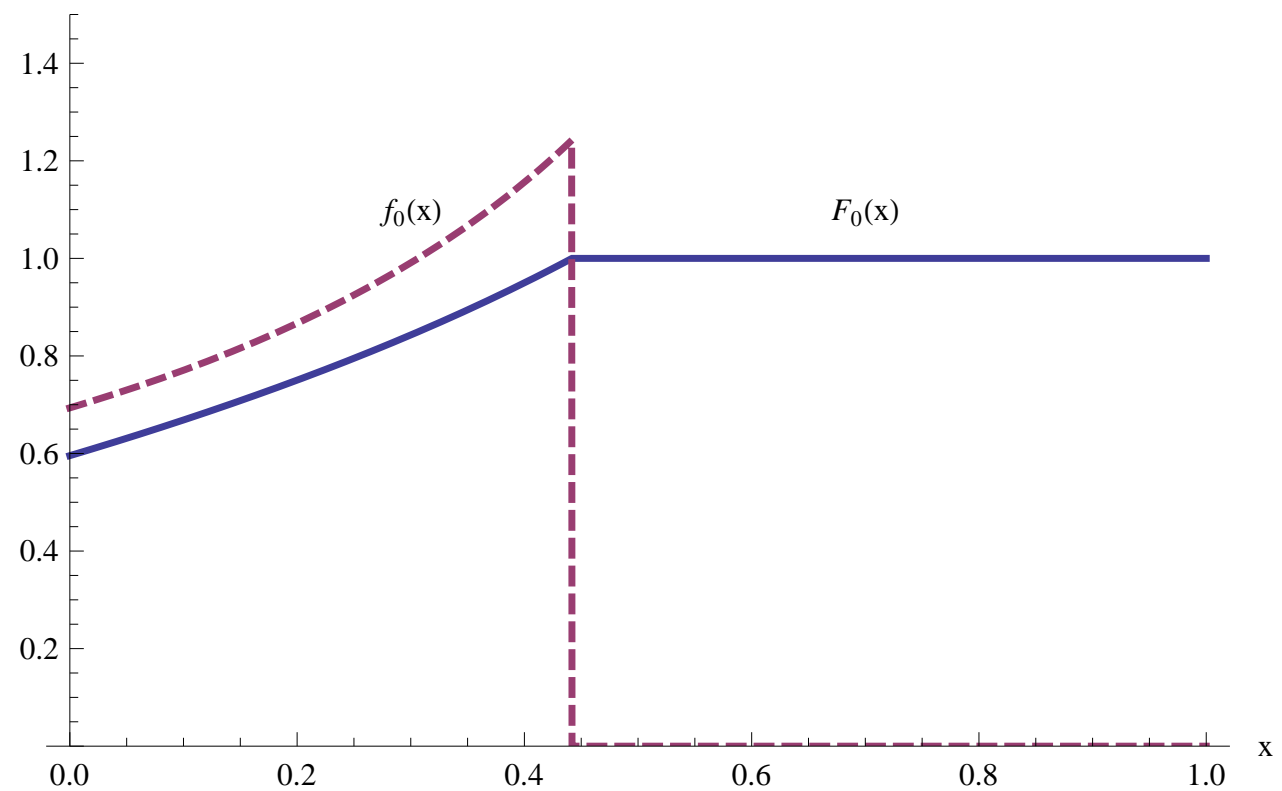

Figure 1: An optimal distribution: The cumulative distribution $F_{0}$ and the density function $f_{0}$.

Lemma 3. Let $\gamma_{0}$ be the root of the equation $1-3 \gamma-(1-\gamma) \ln (1-\gamma)=0$ lying between zero and one $(\approx 0.442)$. Also let

$$
F_{0}(x)= \begin{cases}1-\frac{1}{1+\gamma_{0}} \ln \frac{1-x}{1-\gamma_{0}}, & x \in\left[0, \gamma_{0}\right] \\ 1, & x \in\left(\gamma_{0}, 1\right],\end{cases}
$$

and $\beta_{0}=1 /\left(1+\gamma_{0}\right) .\left(F_{0}, \beta_{0}, \gamma_{0}\right)$ is an optimal solution to $(\mathcal{Q})$. The value of the objective function is then $\gamma_{0} /\left(1-\gamma_{0}\right) \approx 0.792$.

Proof. We will eliminate variables by using the constraints and optimize the objective function. The constraint (11) is rewritten as

$$
F(0)+\int_{0}^{a} f(x) d x+\int_{a}^{\gamma} x f(x) d x-\beta(1+a)=0 .
$$

Differentiating with respect to $a$ and solving the differential equation, we obtain

$$
f(a)=\frac{\beta}{1-a} .
$$

Plugging (13) back in (12), we have

$$
F(0)-\beta(1+\gamma)-\beta \ln (1-\gamma)=0 .
$$

On the other hand, it follows by (10) and (13) that

$$
F(0)-\beta \ln (1-\gamma)-1=0 .
$$

Eliminating $F(0)$ yields

$$
\beta=\frac{1}{1+\gamma}
$$


The objective function (9) is thus simplified as

$$
\frac{1-\gamma-\ln (1-\gamma)}{1+\gamma}
$$

Differentiating with respect to $\gamma$, we have

$$
-\frac{1-3 \gamma-(1-\gamma) \ln (1-\gamma)}{(1-\gamma)(1+\gamma)^{2}}
$$

Solving the equation $1-3 \gamma-(1-\gamma) \ln (1-\gamma)=0$, we get a single root $\gamma=\gamma_{0}(\approx 0.442)$ from the interval $[0,1]$. The function (15) changes its sign from negative to positive at $\gamma=\gamma_{0}$. Therefore, (14) achieves a minimum $\gamma_{0} /\left(1-\gamma_{0}\right)$ at $\gamma_{0} . F_{0}$ is obtained by integrating (13).

\section{Confirmation of the Sufficient Condition}

We confirm that the solution obtained above is an optimal solution to $(\mathcal{P})$ as well.

Lemma 4. $\left(F_{0}, \beta_{0}\right)$ given in Lemma 3 is an optimal solution to $(\mathcal{P})$.

Proof. It is easy to check the feasibility of $\left(F_{0}, \beta_{0}\right)$. We will show that for $\left(F_{0}, \beta_{0}\right)$, one can find $\lambda, \mu$, and $\nu$ which satisfy the conditions of Lemma 2. Although it is certainly enough for completing the proof just to present such $\lambda, \mu$, and $\nu$, we will also mention how to derive them.

We begin with deriving a sufficient condition for (5). Substituting $\eta=0$ into (5) yields

$$
-J(H)=\lambda A(H)+\int_{0}^{1} \mu(x) B(H)(x) d x-\int_{0}^{1} \nu(x) d H(x), \forall H \in \mathrm{ND}[0,1] .
$$

We change the order of the integration of the second term on the right-hand side. Then we obtain

$$
\int_{0}^{1}\left(x+\lambda+\int_{x}^{1} \frac{\mu(a)}{1+a} d a+x \int_{0}^{x} \frac{\mu(a)}{1+a} d a-\nu(x)\right) d H(x)=0, \forall H \in \mathrm{ND}[0,1] .
$$

A sufficient condition for (16) is that: For all $x \in[0,1]$,

$$
x+\lambda+\int_{x}^{1} \frac{\mu(a)}{1+a} d a+x \int_{0}^{x} \frac{\mu(a)}{1+a} d a-\nu(x)=0
$$

holds. On the other hand, a sufficient condition for (5) with $H(x)=0(\forall x \in[0,1])$ and arbitrary $\eta$ is

$$
1-\int_{0}^{1} \mu(x) d x=0 .
$$

Let us substitute $F_{0}$ for $\bar{F}$ in (7). Please recall that $f_{0}(a)>0$ for $a \in\left[0, \gamma_{0}\right]$. To satisfy (7) and (8), we can choose $\nu(a)=0$ for $a \in\left[0, \gamma_{0}\right]$. In turn, note that the equality sign of (3) with $\left(F_{0}, \beta_{0}\right)$ holds strictly for $a \in\left(\gamma_{0}, 1\right]$. Similarly, it is sufficient for (6) and (8) that $\mu(a)=0$ for $a \in\left(\gamma_{0}, 1\right]$.

Employing (17), we determine $\mu$ and $\nu$ on the remaining intervals for each. Plugging $\nu(a)=0$ $\left(a \in\left[0, \gamma_{0}\right]\right)$ into (17) yields an integral equation. By solving it, we obtain

$$
\mu(a)=\frac{1+a}{(1-a)^{2}}, a \in\left[0, \gamma_{0}\right] .
$$


Here the constant of integration is determined by (18). This also gives

$$
\lambda=-\frac{\gamma_{0}}{1-\gamma_{0}} .
$$

again by (17). Besides, (17) for $x \in\left(\gamma_{0}, 1\right]$ determines

$$
\nu(a)=\frac{a-\gamma_{0}}{1-\gamma_{0}}, a \in\left(\gamma_{0}, 1\right] .
$$

$\nu$ is indeed continuous even at the point $a=\gamma_{0}$. It is confirmed that the obtained $\lambda, \mu$, and $\nu$ satisfy the conditions of Lemma 2 .

\section{References}

[CNS10] C. Chung, T. Nonner, and A. Souza. SRPT is 1.86-competitive for completion time scheduling. In Proc. SODA '10, pages 1373-1388, 2010.

[FIS11] H. Fujiwara, K. Iwama, and Y. Sekiguchi. Average-case competitive analyses for one-way trading. J. Comb. Optim., 21(1):83-107, 2011.

[Lue69] D. G. Luenberger. Optimization by vector space methods. John Wiley \& Sons Inc., New York, 1969.

[PSW98] C. A. Phillips, C. Stein, and J. Wein. Minimizing average completion time in the presence of release dates. Math. Program., 82:199-223, 1998.

[Roy88] H. L. Royden. Real Analysis. Prentice Hall, third edition, 1988.

[RS90] F. Riesz and B. Sz.-Nagy. Functional Analysis. Dover Publications, Inc., New York, 1990.

[Sit10] R. Sitters. Efficient algorithms for average completion time scheduling. In Proc. IPCO '10, pages 411-423, 2010. 